\title{
La fuente del Heracleo de Gades en la ciencia antigua
}

\author{
Pamina Fernández CAMACHO \\ Universidad de Cádiz \\ pamina.fc@gmail.com
}

Recibido: 3-09-2012

Aceptado: 20-12-2012

\section{RESUMEN}

La digresión sobre el comportamiento aparentemente anómalo de una fuente del Heracleo gaditano, y su relación con los ritmos de las mareas, ocupa una parte considerable de la descripción de la isla contenida en los apartados 3.5.3-10 de la obra del geógrafo de Amasía. En él se exponen teorías de historiadores, geógrafos y científicos que habían tratado el tema anteriormente, a las que Estrabón, a su vez, añade sus propias opiniones. El estudio de este debate nos permitirá arrojar una luz sobre la importancia de esta fuente en la fenomenología acuática de la época (a pesar de su ausencia en los catálogos paradoxográficos conservados), así como sobre el trasfondo científico de las explicaciones propuestas.

Palabras clave: Ciencia griega, Estrabón, Gades, Polibio, Posidonio.

\begin{abstract}
The digression about the apparently anomalous behaviour of a fountain located at the Herakleion in Gades, and how it relates to the movements of the tide, takes a large part of the island's description contained in paragraphs 3.5.3-10 of the Amaseian geographer's work. Theories from historians, geographers and scientists who had written on that subject are quoted, together with Strabo's personal opinions. A study of this debate will give us an insight on both the importance of that fountain in the literature of water phenomenology of the time (in spite of its absence from extant paradoxographic works), and the scientific background of the proposed explanations.
\end{abstract}

Keywords: Gades, Greek Science, Polybius, Posidonius, Strabo.

\section{SUMARIO}

1. Introducción: El texto en su contexto. Las fuentes. 2. Cuestiones científicas. 3. Conclusiones. 4. Bibliografía. 


\section{INTRODUCCIÓN: EL TEXTO EN SU CONTEXTO. LAS FUENTES}

En la Geografía de Estrabón, el espacio dedicado a Gadira es considerable. La mayor parte del capítulo dedicado a las islas de la Península Ibérica se encuentra centrado en este lugar, geográficamente diminuto pero de gran importancia en el mapa comercial, político y - sobre todo- simbólico de la época. Allí se localizaba una importante colonia fenicia aliada de Roma, conocida y citada desde la época arcaica como límite del extremo occidental del mundo en asociación con las llamadas Columnas de Hércules, que según ciertas fuentes se encontraban en la propia isla ${ }^{1}$.

Por ello, no es de extrañar que buena parte del pasaje estraboniano esté consagrado a una serie de digresiones referentes a cuestiones de interés geográfico, científico, mitológico o botánico relacionadas con la problemática de los confines en general, y el Extremo Occidente en particular. Así, 3.5.4 recuerda la identificación de la isla con la legendaria Eritía por parte de Ferécides, y la consiguiente localización en ella del mito de Gerión, recogido por primera vez en la Teogonía de Hesíodo y situado en la zona - con propósitos coloniales - a partir de la Gerioneida de Estesícoro². 3.5.5-6 refleja un largo debate sobre la localización exacta de las Columnas de Hércules, el hito occidental más importante en la geografía griega y romana. Dos opiniones se imponen: aquélla, defendida por «la mayoría de los griegos», que identifica las Columnas con accidentes geográficos del Estrecho, y una segunda que las identifica con Cádiz, ya sea con las columnas del templo de Heracles-Melqart (como creen los iberos, los libios, y el filósofo Posidonio), o con el mismo lugar donde se establece la ciudad, como parece desprenderse de una leyenda de fundación narrada por los propios gaditanos ${ }^{3}$. 3.5.10, por su parte, documenta la existencia de un árbol de características maravillosas descrito por Posidonio, y que posiblemente deba identificarse con los misteriosos árboles de Gerión mencionados por Pausanias y Filóstrato ${ }^{4}$; por lo demás, la fauna y la vegetación «fuera de lo común» son propios de la geografía de los confines del mundo 5 .

Lo mismo sucede con los fenómenos relacionados con las mareas del océano, que bañaba los límites de la tierra habitada. Cádiz, la ciudad oceánica por antonomasia, era el destino de científicos que, como Posidonio y el Apolonio de Tiana biografiado por Filóstrato, acudían a observar las mareas, mucho más conspicuas allí que en el Mediterráneo. Así fue como una fuente relacionada con el culto local, de la que se decía que presentaba un comportamiento exactamente inverso al de éstas, adquirió una gran importancia para la erudición de la época, tanta que Estrabón le dedica la digresión más extensa del pasaje (3.5.7-3.5.9).

${ }^{1}$ Cf. Str. 3.5.5-6, Philostr. V.A.5.5. Sobre la utilización de Cádiz como límite, cf. Pi. N.4.69, Str. 3.5.5-6, $R G$ 26.9, entre otros.

${ }^{2}$ Hes. Th. 290 y ss, Stesich. Fr. 7 PAge, SLG (=Str. 3.2.11.)

3 También podemos observar versiones «intermedias», o de transición en Ps.-Scyl 1-2; 111, cf.

Peretti (1979: 154-169), o Philostr.V.A.5.1.

${ }^{4}$ Paus. 1.35.8, Philostr. V.A.5.5.

5 Cf. Romm (1992), para un estudio de la geografía de los confines. 


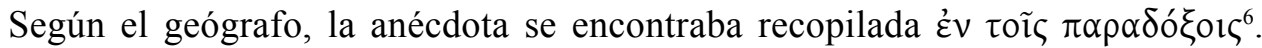
A pesar de esta afirmación, no se conserva rastro alguno de ella en la literatura paradoxográfica propiamente dicha, ni en sus largos catálogos de fuentes, lagos y mares fabulosos ${ }^{7}$. Sí forma parte de un catálogo de carácter paradoxográfico inserto en un texto que pertenece a otro género: la recopilación de fenómenos inusuales relacionados con el agua del libro II de la Historia Natural ${ }^{8}$ de Plinio. En esta obra, como se estila entre los autores latinos, la paradoxografía, cuyo repertorio procede originalmente de los textos científicos, es utilizada a su vez para documentar obras de carácter científico?.

Pero es en la Geografía de Estrabón donde el $\pi \alpha \rho \alpha ́ \delta \circ \xi_{o v}$ adquirirá un puesto de pleno derecho en la tradición científica. Tanto la amplitud del pasaje como los nombres de autores helenísticos de primera fila que en él se citan y la divergencia de sus opiniones prueban la existencia de un debate importante, que podemos contraponer

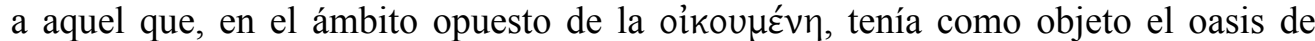
Amón. La fuente - o fuentes - que la tradición situaba en dicho lugar también se relacionaba con un centro religioso de importancia, el oráculo donde Alejandro fue proclamado inmortal. Más afortunado en lo que respecta a la conservación de los tex-

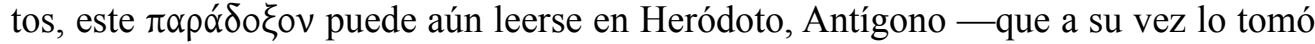
de Calímaco, que lo había hallado en la obra de Aristóteles-, y más tarde Diodoro, Arriano, Lucrecio, Quinto Curcio y Plinio ${ }^{10}$, buena parte de los cuales recibieron la noticia a través de la tradición biográfica de Alejandro. El pasaje de Estrabón sobre el Heracleo, por su parte, resume un debate en el que, además de él mismo, han participado al menos Sileno, Artemidoro, Polibio y Posidonio, todos ellos pioneros en el conocimiento griego de Occidente como Heródoto y los historiadores de Alejandro Magno lo fueron del de Oriente. Asimismo contiene la ya mencionada referencia a los $\pi \alpha \rho \alpha ́ \delta \circ \xi \alpha$ desconocidos donde Estrabón habría leído la noticia, y una teoría sobre las mareas propuesta por Atenodoro que puede o no haber guardado relación con la fuente en su origen. El esquema completo del debate, tal y como lo describe Estrabón, es el siguente (cf. KIDD, 1988: 768 ss):

(1) Estrabón cita las observaciones de Polibio sobre la fuente del Herakleion de Gadira, que se comporta de forma inversa con respecto a las mareas. También añade la explicación que ofrece Polibio a tal fenómeno: la marea alta bloquea la salida de las corrientes de aire subterráneas, que a su vez se desplazan al interior y bloquean los conductos de la fuente.

6 Str.3.5.7.

7 En Pajón LeYra (2009: 510-511), se propone la identificación de esta fuente con Isígono de Nicea (ca. 150-100 a.C), paradoxógrafo de renombre que se ocupó de cuestiones hidrográficas en su obra $\mathrm{Ca}$ tálogo de fenómenos increíbles, fragmentos de la cual se conservan en el Paradoxógrafo Florentino. Cf. GiANNINI (1965).

${ }^{8}$ Plin. nat.2.212-238. También cf. Plin. nat.31.1-72 para un catálogo complementario sobre las utilidades del agua, también frecuentemente de carácter paradoxográfico.

9 Pajón Leyra (2009: 503), Schepens-Delcroix (1996: 433-439).

${ }^{10}$ Hdt. 4.181, Call. Fr. 28 Giannini (=Antig. Mir. 144), Arist. Fr. 531 Rose, D.S. 17.50.4-5, Arr. An. 3.4, Lucr. 6.848 ss., Curt. 4.7.22, Plin. nat. 2.228. Cf. PAJón LeYra (2009: 294). 
(2) Estrabón menciona que Artemidoro ha criticado la explicación de Polibio y ofrecido la suya propia, «recordando» o «trayendo a colación» ( $\mu \nu \eta \sigma \theta \varepsilon i \varsigma)$ la opinión del historiador Sileno. Pero no considera oportuno repetir tales teorías, tachando desdeñosamente a estos dos autores de $i \delta i \omega ́ \tau \eta \uparrow$, es decir, profanos

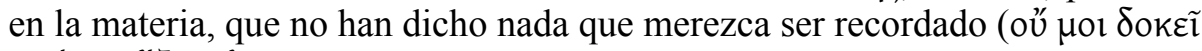

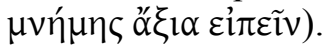

(3) Posidonio, por su parte, ha declarado que esta historia, que también se encuentra en los $\pi \alpha \rho \alpha ́ \delta \circ \xi \alpha$ que el mismo Estrabón ha leído, es falsa. Después de investigar el tema durante su estancia en Cádiz, concluye que hay dos pozos en el Herakleion y uno en la ciudad, y que al retirar agua de ellos durante el día baja su nivel, mientras que, por la noche, cuando no se utilizan, éste se restablece. Los gaditanos habrían atribuido esta subida a una relación de ờ $v \imath \imath \alpha^{\prime} \theta \varepsilon l \alpha$ de la marea con el pozo, porque la marea baja coincidía frecuentemente con esta hora de la noche (y el rumor habría llegado hasta los otros autores, que le habría prestado oídos en lugar de observar el fenómeno por sí mismos). Como bien indica Estrabón, la marea baja coincidiría con esa hora de la noche tanto como la alta, y los habitantes de Gadira deberían haber sido verdaderamente estúpidos para no darse cuenta (cuando, sin embargo, habían sido capaces de observar los ciclos anuales de la marea de forma lo bastante satisfactoria como para merecer el crédito del propio Posidonio).

(4) Estrabón prefiere dar crédito a la teoría de Polibio, pero añade dos explicaciones complementarias. La primera parece de su propia cosecha (aunque no es ésta la opinión de $\left.\mathrm{Kidd}^{11}\right)$, pues no cita a ningún otro autor y la contraposición $(\mu \dot{v} v$ ....غ̇...) la separa de la opinión de Polibio. Según esta explicación, el agua de la marea impregnaría la tierra y empaparía las paredes de los conductos de la fuente, disminuyendo su consistencia y permitiendo que el agua se escapara por una serie de «goteras» que impedirían al chorro llegar intacto al final de su camino. La otra explicación se atribuye a Atenodoro, en el marco de una teoría sobre las mareas, aunque ignoramos si nuestra fuente ocupaba algún lugar en ella. Para Atenodoro (como para Mela, Filóstrato o el propio Estrabón ${ }^{12}$ ) las mareas se corresponden con movimientos de inhalación y exhalación, como la respiración de los seres vivos. El agua subterránea tiene dos caminos para salir a la superficie: o bien a través de la fuente, o a través de conductos submarinos que la unirían al mar. Cuando el mar inhala, el movimiento arrastraría el agua a estos conductos submarinos, y ésta ayudaría a provocar la subida de la marea. Luego volvería a los conductos de la fuente, restableciendo su flujo por un tiempo.

Detengámonos un instante en la información que proporciona el pasaje sobre las diversas fuentes. En primer lugar, Estrabón menciona a Polibio (ca. 200-118 a.C), historiador griego del círculo de Escipión y principal representante de la llamada historiografía pragmática, basada en hechos político-militares ${ }^{13}$. Esta $\pi \rho \alpha \gamma \mu \alpha \tau \imath \kappa \tilde{\eta} \varsigma$

\footnotetext{
${ }^{11}$ KIDD (1988: 770).

${ }_{12}$ Mela. 3.1-2, Philostr. V.A. 5.2.; Str. 1.3.8.

13 Sobre el método de Polibio, el trabajo más importante hasta la fecha es PéDECH (1964).
} 
iotopíac, según el propio Polibio, debía construirse a partir de tres componentes: el estudio de los documentos escritos, el análisis de los acontecimientos políticos, y la contemplación ( $\theta \dot{\varepsilon} \alpha v)$ de los lugares, las distancias, y las «pecularidades de la tierra y

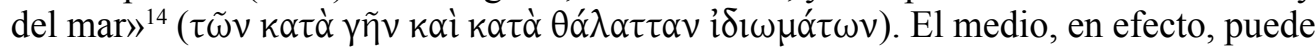
influir directamente en los hechos históricos: el conocimiento de la topografía y las distancias es esencial en la guerra (3.48.4), y las características de un lugar podían afectar la suerte de sus habitantes empujándolos a la molicie y a la decadencia (7.1.1), conviertiéndolos en salvajes e incivilizados (4.21.1-6) o haciéndolos objeto del deseo de potenciales conquistadores $(1.7 .2)^{15}$. No obstante, y a pesar de la importancia que él mismo otorgaba al conocimiento directo o «contemplación» del terreno, Polibio admitía que era imposible conocer personalmente todos los lugares de los que trataba. Por ello, a veces había que contentarse con «obtener impresiones e ideas de estos (lugares) tan próximas a la verdad como fuera posible. $\left.{ }^{16}\right\rangle$

Estas consideraciones geográficas se encuentran diseminadas por los distintos libros de su Historia, aunque, según Pédech, Polibio fue interesándose cada vez más en la geografía como ciencia a partir de los viajes y las investigaciones sobre el terreno que llevó a cabo para su obra histórica, sobre todo cuando éstos le condujeron hacia las zonas occidentales del mundo ${ }^{17}$. Estas muestras de interés culminaron en la elaboración del libro 34, un excurso general sobre geografía. En este libro «especializado», a partir de los fragmentos que conservamos, nuestro historiador parece haber descrito los distintos mares, continentes, regiones, ríos y pueblos del mundo, aventurado unas medidas para el globo terrestre, expuesto su propia opinión sobre la verdad de la geografia homérica - al igual que Estrabón - y polemizado con otros geógrafos. A él se atribuye asimismo la descripción de la fuente del templo de Heracles-Melqart de Cádiz ${ }^{18}$.

Polibio visitó la Península Ibérica en persona, bajo la protección de las águilas romanas. En sus propias palabras, desafió los peligros que suponía viajar katà $\Lambda \mathrm{i} \beta u ́ \eta v$

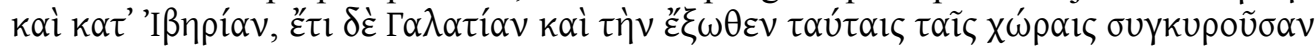
$\theta \alpha ́ \lambda \alpha \tau \tau \alpha \nu$ (3.59.7). Esta asociación de mares y extremos apunta a una visita a Gadira, estrechamente relacionada con ambos conceptos en el imaginario griego y, que, de hecho, describió en una parte perdida de su obra ${ }^{19}$. Tal visita debería haberle permitido contemplar la fuente fabulosa con sus propios ojos, y con ello asegurarse de la veracidad del fenómeno. Posidonio, no obstante, le acusa de haberse dejado llevar por las

${ }^{14}$ Plb. $12.25 \mathrm{e}$.

15 Estos y otros ejemplos en Clarke (1999: 77-128).

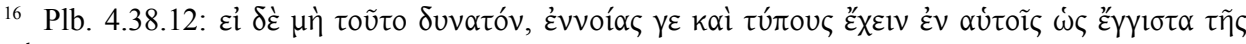
$\dot{\alpha} \lambda \eta \theta \varepsilon i ́ \alpha \varsigma$

17 PÉDECH (1964: 555): «Jusqu'ici la géographie de Polybe se mêle étroitement à l'histoire: toutes les indications topographiques, distances, relief du sol, position et situation des villes, ressources locales, servent à expliquer les évenements et entrent dans le faisceau des causes et des modalités (...). Des voyages et la découverte d'horizons nouveaux vont élargir et enrichir sa conaissance du monde; mais en même temps sa géographie perdra le contact des préoccupations politiques; l'homme d'État s'effacera devant le voyageur qui, ébloui des perspectives révélées, cultivera la géographie por elle même avec la curiosité et le désintéressement du savant.»

${ }^{18}$ PÉDECH (1956: 3-24).

${ }^{19}$ Plin. nat. 4.119. 
creencias erróneas de los gaditanos. Si Polibio estuvo allí, no se había molestado en observar el funcionamiento de la fuente, sino que se había limitado a recoger historias sobre ella, al contrario que él, que llevó a cabo las más exhaustivas investigaciones en persona. Un cierto sentimiento de rivalidad científica - la crítica del predecesorparece subyacer en este discurso.

También otras rivalidades se desprenden del pasaje. Aunque es el primer autor mencionado por Estrabón, Polibio no pudo ser el primero en escribir sobre la fuente del Heracleo. Este honor debió recaer, o bien en Sileno de Caleacte, historiador y acompañante de Aníbal en sus campañas ${ }^{20}$, o tal vez en la «bestia negra» de Polibio, Píteas de Marsella.

Que Sileno habló de Cádiz en su obra, hoy perdida por completo, lo sabemos gracias a este pasaje de Estrabón y a un pasaje de Plinio, en el que se le atribuye la información de que la isla donde se encontraba antiguamente la ciudad de Cádiz era llamada Afrodisias $^{21}$. Por desgracia, no podemos decir mucho más de su obra, sus ideas, o su calidad como historiador. Pudo haber estado en el Heracleo con Aníbal ${ }^{22}$, y visto la fuente con sus propios ojos, o haber conocido su existencia de boca del propio general cartaginés o de alguien de su ejército, pero su teoría es despreciada hasta el punto que Estrabón ni

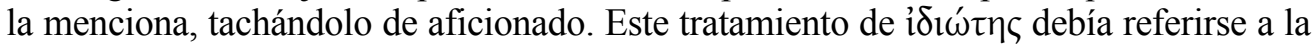
ignorancia de Sileno en cuestiones científicas, lo que lo deja al nivel de Artemidoro en este pasaje - geógrafo ya criticado por Estrabón a causa de su credulidad con respecto a las historias de oi $\pi \circ \lambda \lambda o^{23}$ - y lo oponía a Polibio, con cuya opinión está de acuerdo Estrabón, así como a Atenodoro y Posidonio, científicos de renombre. ¿Pero era ésta la opinión que tenía el propio Estrabón de Sileno, o la había heredado de sus antecesores?24

Es posible que Polibio ya lo hubiese criticado en su propia obra. Se ha señalado el afán del megalopolitano por desacreditar a sus competidores por el título de primero en observar el extremo occidental del mundo ${ }^{25}$. Esta intención resulta evidente cuando declara que los autores anteriores a él no pudieron obtener información fiable de las regiones extremas debido a su lejanía y barbarie; ahora que él, Polibio, lo había conseguido gracias a las conquistas de los romanos, las erróneas percepciones anteriores podrían al fin ser corregidas (3.58-59). Si alguien pretendía habérsele adelantado en este propósito, tenía que vérselas con sus afiladas críticas. A lo largo de su obra mantuvo agrias polémicas con Timeo, autor de una historia de Occidente que había alcanzado un gran éxito entre el público romano de la época ${ }^{26}$, y al que acusaba, entre otras cosas, de no haber observado en persona las cosas de las que escribía (12.3-16, 23-28a).

${ }^{20}$ FGH $175 \mathrm{~T} 2$ (=NeP. Hann. 13.3): huius (sc. Hannibalis) belli gesta multi memoriae prodiderunt, sed ex his duo, qui cum eo in castris fuerunt simulque vixerunt, quamdiu Fortuna passa est, Silenus et Sosilus Lacedaemonius.

${ }^{21}$ Plin. nat. 4.120.

${ }^{22}$ Liv. 21.21.

${ }^{23}$ Str.3.1.4-5.

${ }^{24}$ KIDD (1988: 769): «This doxographical note may also have been in Posidonius, although Strabo brings in Artemidorus and Silanus merely to contrast them with Posidonius.»

${ }^{25}$ WaLbank (1972: 52).

${ }^{26}$ VATtuone (2002: 177-232). 
Otro de los rivales de Polibio era Píteas de Marsella, el primer viajero que exploró el Atlántico desde Gadira. A éste lo acusa de mentiroso, y de no haber estado donde decía (34.5), y Estrabón se hace eco de esta opinión negativa a lo largo de su obra. Lo cual resulta más que significativo, pues Píteas, que elaboró el tratado pionero sobre las mareas Sobre el Océano y visitó Gadira, pudo haber sido la fuente primera y original de toda esta historia de la fuente del Heracleo, relacionada en el texto de Estrabón con un debate sobre las mareas. Según Pajón Leyra ${ }^{27}$, sin embargo, existen motivos para pensar que éste no fue el caso, pues ¿habría reconocido Polibio, que disputaba la credibilidad de los viajes de Píteas, que éste había transmitido un fenómeno verdadero observado en la misma Gadira? En cualquier caso, desgraciadamente no se han conservado testimonios que ayuden a clarificar el asunto ${ }^{28}$.

Por otra parte, Polibio no es el único en criticar a sus antecesores para reivindicar su posición. Artemidoro, — que también visitó la Península y Cádiz—29 ${ }^{29}$, criticó la explicación del fenómeno del megalopolitano en su propia Geografía. Y por último, Posidonio, que viajó a la Península y pasó un mes en Cádiz, no perdió tampoco la oportunidad de formular sus propias críticas. En este texto declara falsa $(\psi \varepsilon \cup \delta \tilde{\eta})$ la historia de la fuente transmitida por todos ellos, y los acusa de dejarse guiar por rumores infundados. Sólo él ha contemplado la verdadera realidad, y no sólo con respecto

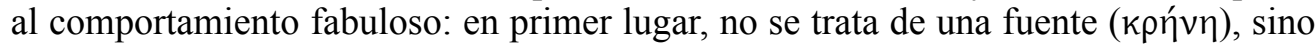
de un pozo ( $\varphi \rho \varepsilon ́ \alpha \rho)$; no es uno, sino dos, uno más grande que el otro, y además hay un tercero en la ciudad. Más allá de la acusación directa, la misma acumulación de detalles parece apuntar a un propósito polémico. Sin embargo, su opinión no halló crédito por parte de Estrabón, que critica su lógica y además aprovecha para introducir

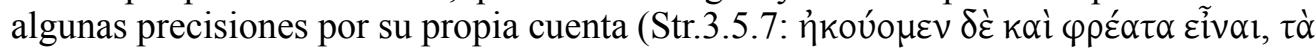

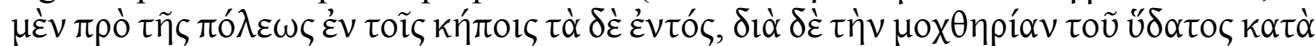

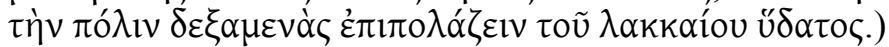

Acabamos esta recapitulación de las fuentes con una mención a Atenodoro. Filósofo estoico en la época de Estrabón ${ }^{30}$, pudo muy bien haber participado en el debate, mencionando el fenómeno en su obra perdida sobre las mareas. Si esto es así, su argumento es expuesto por Estrabón en (4), y se encuentra entre los tres que el geógrafo

${ }^{27}$ PAJÓN LeYra (2009: 510, n.1417.)

${ }^{28}$ Otras opiniones sobre las razones de Polibio para desacreditar a Píteas, en el marco de una oposición entre "geógrafos científicos» y «geógrafos políticos», sensibles a los intereses del poder romano, cf. S. Bianchetti, «Eutimene e Pitea di Massalia: geografia e storiografia», en VATTUONE (2002: 439-485), y Bianchetti (1997: 80): «La polemica dello storico (Polibio) nei confronti di Pitea, il cui viaggio a Thule è giudicato impossibile, assume l'aspetto di una rivendicazione della superiorità delle imprese romane, in particolare quelle di Scipione Emiliano, rispetto à tutte le altre», así como BIANCHETTI (2008: 31): «il superamento della pura dimensione odologica, realizzato dal Massaliota attraverso la messa a punto di coordinate astronomiche e tradotto nella carta di Eratostene, che utilizava i dati empirici all'interno di un reticolo costruito su base scientifica, comporta una vera e propria revoluzione, destinata ad essere "dimenticata» proprio per il carattere destabilizzante che la nuova immagine del mondo implicava in rapporto a precisi contesti politici: Polibio, Artemidoro, Strabone, Mela, Plinio operano, infatti, "correzzioni" dietro le quali non è difficile cogliere (...) motivazioni di ordine politico e che giustificano la resistenza opposta dai geografi storici ad accogliere la carta degli scienziati.»

${ }^{29}$ Marcian. Epit.Menipp., 3.31-35 (GGM 1. 566).

${ }^{30}$ FGH 746 T.1 (=Str.14.5.14). 
considera plausibles (junto con el de Polibio y el enunciado por él mismo). Otra opción sería entender que Estrabón ha utilizado una teoría que se encontraba en la obra sobre las mareas de Atenodoro para buscar una solución al problema de la fuente, sin que Atenodoro hubiera tratado el tema directamente.

\section{CUESTIONES CIENTÍFICAS}

Tanto el resumen que hace Estrabón de las teorías referentes a la fuente del Heracleo como su propia opinión sobre el fenómeno pertenecen, pues, al panorama científico de la época. Como tales, se basan en una serie de conceptos enraizados en la ciencia natural griega. A continuación, pondremos de relieve algunas cuestiones relacionadas con la interpretación científica del texto.

En primer lugar, observamos que el fenómeno del comportamiento de la fuente

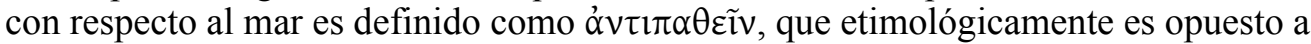

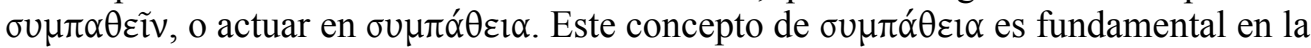
escuela estoica, a la que Estrabón se enorgullece de pertenecer, y de la que Posidonio, su probable fuente para parte de este pasaje, era uno de los principales representantes.

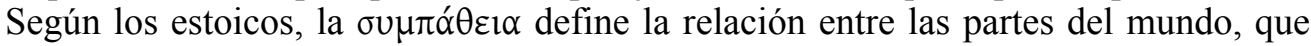
para ellos equivale a la unión de los miembros de un ser vivo más que a una simple aglomeración de unidades como un coro o un ejército:

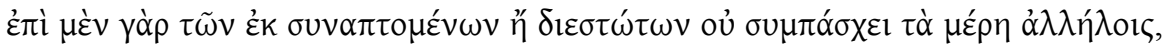

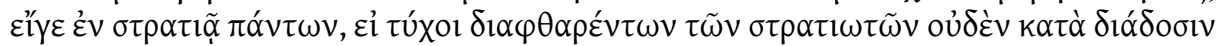

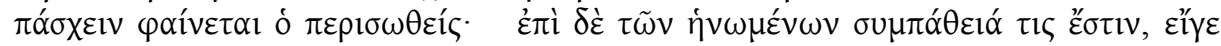

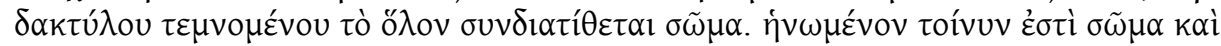
ó kó $\mu \operatorname{o\zeta }^{31}$.

Una sustancia ligera hecha a partir de fuego y aire, el pneuma, permea el universo entero haciendo posible esta relación.

Por otra parte, una confirmación empírica de esta simpatía entre las partes del mundo venía suministrada por la relación de la luna con las mareas. Esta teoría será precisamente expuesta por Estrabón a continuación de este pasaje, así como, veremos, la conexión entre dicho fenómeno y el estado de los animales y seres humanos, ya observado por Aristóteles según Plin.nat.2.220. Por ello, Kidd establece una relación

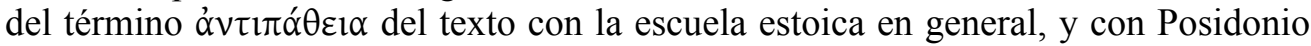
en especial ${ }^{32}$. No creemos, sin embargo, que tal relación esté del todo justificada. El término ớ $v \tau \imath \alpha_{\alpha} \theta \varepsilon \imath \alpha$, con el significado específico que presenta en este pasaje, no forma parte del vocabulario técnico de los estoicos cuyas obras se nos han conservado (fuera de este contexto sólo aparece en Posid. Fr 309A THEILER, fragmento, por cierto no recogido por Jacoby en su $F G H$.) Su utilización aquí obedece a una necesidad puntual de definir un fenómeno de carácter anómalo, un $\pi \alpha \rho \alpha ́ \delta \circ \xi o v$, lo que chocaría

\footnotetext{
${ }^{31}$ S.E. M. 9.80 .

${ }^{32}$ KIDD (1988: 768-769).
} 
con su pertenencia a un modelo cosmológico amplio, donde una tal oposición al orden

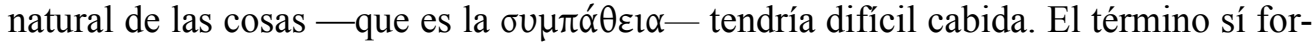
ma parte, no obstante, del vocabulario mágico, un campo propicio al establecimiento de actuaciones variadas de unas sustancias sobre otras. El pitagórico egipcio Bolo de Mendes, que escribió una obra sobre la simpatía y la antipatía de las piedras, declaró que la naturaleza complaciéndose en otra naturaleza era $\sigma u \mu \pi \alpha ́ \theta \varepsilon 1 \alpha$, mientras que la naturaleza conquistando a otra naturaleza era ó $v \tau \imath \pi \alpha ́ \alpha \varepsilon ı \alpha^{33}$.

A continuación, Estrabón procede a introducir la opinión de Polibio sobre el fenómeno. Según el testimonio de este texto, el megalopolitano habría tratado de explicar la anomalía a través de la actuación directa de unos elementos sobre otros: durante la marea llena, el agua del mar corta el camino al aire, y éste, a su vez, penetra en las «venas» del manantial y corta el camino al agua de la fuente. Esta teoría implica al menos dos presupuestos: uno de ellos es la teoría de los cuatro elementos formulada por primera vez por Empédocles de Agrigento $^{34}$, y que conocería un gran éxito en la ciencia griega, siendo adoptada, con ciertas variantes, tanto por la escuela platónica como por la aristotélica y también por el estoicismo. Todos los fenómenos se explican a través de la interacción de unos elementos sobre otros. Y en estas explicaciones el aire, en especial, ocupa un lugar destacado. Anaxímenes lo creía la esencia del cosmos, y origen de todos los demás elementos. Al ser el elemento más sutil, y que puede encontrarse siempre en los intersticios dejados por los otros elementos, es el que suele poner los procesos naturales en movimiento. Como indica Séneca, a propósito de su relación con el elemento sólido: Aer continuus terrae est et sic appositus ut statim ibi futurus sit unde illa discesserit ${ }^{35}$. También es el más apto a entrar en tensión, y mover a los otros objetos ${ }^{36}$. Al contrario que los otros dos elementos involucrados, el agua y la tierra, se trata de un elemento considerado activo ${ }^{37}$, y su unión con el fuego constituye el pneuma, agente por excelencia del cosmos estoico ${ }^{38}$.

Por ello es también a través de la presión del aire como se explica un fenómeno tan devastador y que provocaba tal temor y fascinación en la época como el terremoto. El aire, que trata de salir de la tierra, se encuentra bloqueado, y la tensión aumenta hasta que «rompe» la tierra para hallar una salida ${ }^{39}$. En el caso de la fuente del Heracleo, igualmente, el aire es el que tapona la fuente al encontrar cerrado su camino natural hacia la superficie.

El segundo presupuesto de la teoría polibiana es la teoría del encadenamiento de las causas, provocado por la ausencia de vacío. Platón, en su diálogo Timeo, define el proceso de esta manera al referirse al fenómeno de la respiración:

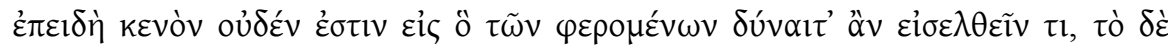

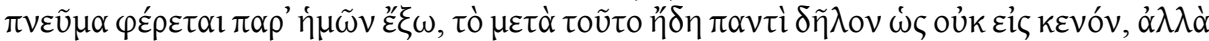

\footnotetext{
${ }^{33}$ IRBY-MASSIE, KeYSER (2002: 236).

${ }^{34}$ Diels (1879: 286-287, 582, 591.)

${ }^{35}$ Sen. nat. 2.6.1.

${ }^{36}$ Sen. nat. 2.8.1.

${ }^{37}$ Gal. de nat. facult. 1.3.8, cf. SAMBURSKY (1959: 5).

${ }^{38}$ SAMBURSKY (1959: 4).

${ }^{39}$ Sen. nat. 6.12-26.
} 


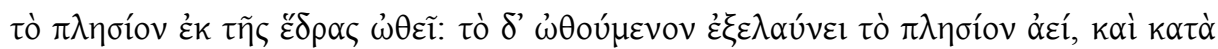

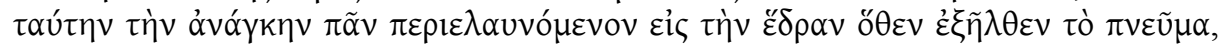

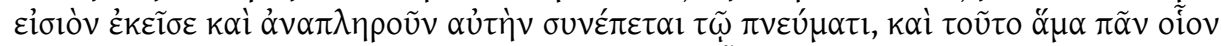

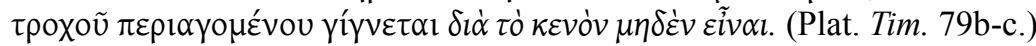

Y, más adelante, aplicando también el concepto a los fenómenos naturales:

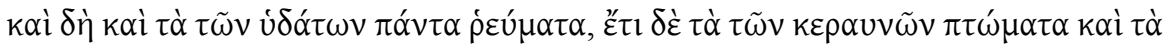

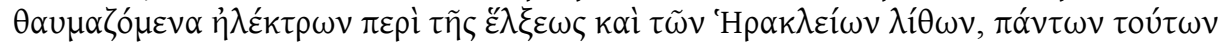

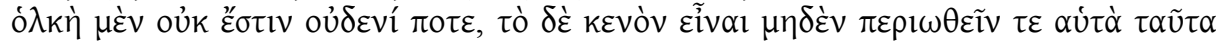

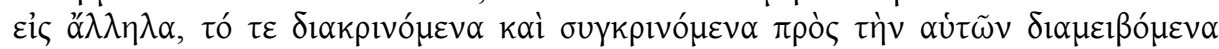

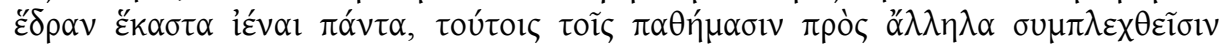

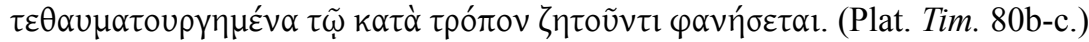

De las dos grandes escuelas surgidas en la época helenística, los estoicos tomaron también el camino de negar el vacío en el interior del cosmos —sí existía, en cambio, en el exterior ${ }^{40}$ _, mientras que los epicúreos, por el contrario, defendían que la materia estaba formada por unos corpúsculos diminutos, los átomos, que se movían a través de él ${ }^{41}$. Según la primera concepción, que es la que presupone nuestro texto, los fenómenos se explican mediante la actuación de unos cuerpos sobre otros en forma de un inexorable efecto en cadena: el mundo es un continuum dinámico donde cada desplazamiento implica a su vez un nuevo desplazamiento. Tal forma de entender el mundo físico conduciría, a su vez, a una concepción determinista de todas las acciones, ya que

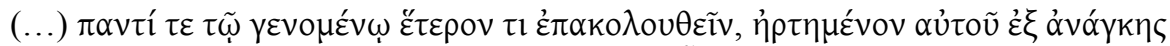

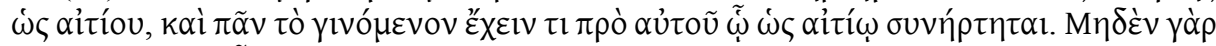

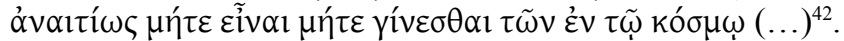

Según la teoría epicúrea, sin embargo, los responsables de los fenómenos serían los choques entre átomos, una visión que se aleja drásticamente de esta tradición científica ${ }^{43}$.

Pasemos ahora a la siguiente teoría, que es la de Posidonio, ya que las de Artemidoro y Sileno son ignoradas por tratarse sus autores de simples «profanos». Posidonio, al contrario que ellos, gozaba de una gran reputación como filósofo y científico, lo cual contrasta de un modo un tanto sorprendente con la opinión que se le atribuye aquí.

Según Posidonio, en efecto, la explicación del fenómeno no es científica en absoluto. Se trata tan sólo de un error de percepción de la gente de la zona, pues la supuesta fuente, que no es sino un pozo, se utiliza para sacar agua, por lo que su nivel va disminuyendo durante el día. De noche, cuando ya no se saca más agua, el nivel aumenta de nuevo, y la opinión popular habría relacionado este momento con la bajada de la marea. La expresión exacta del texto de Posidonio es $\dot{\varepsilon} \pi \varepsilon l \delta \dot{\eta} \delta \dot{\varepsilon} \sigma v \mu \pi i ́ \pi \tau \varepsilon l \kappa \alpha \tau \dot{\alpha} \tau \dot{o} \nu \tau \tilde{\eta} \varsigma$

\footnotetext{
${ }^{40}$ Plu. Stoic.rep. 43 (Mor.1054b), D.L. 7.140.

${ }^{41}$ Lucr. 1.483-634.

${ }^{42}$ Alex.Aphr. Fat. 22. cf. SAMBURSKy (1959: 69).

${ }^{43}$ Lloyd (1973, 28-29).
} 


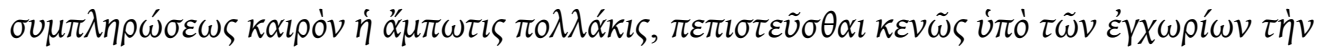

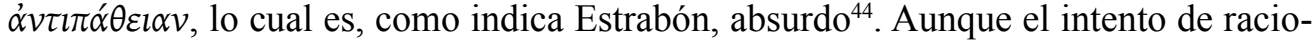
nalización de un fenómeno percibido como anormal es propio de la ciencia estoica, resulta difícil de creer que el científico que estudió las mareas de forma tan meticulosa pudiera haber hecho tal afirmación, por lo que sería más prudente considerar que Posidonio se habría limitado a declarar que los nativos habían establecido esa relación errónea entre la bajamar y la noche, en lugar de establecerla él mismo. Tal versión encajaría con el grueso de la refutación de Estrabón, que critica a Posidonio por haber atribuido tal falta de discernimiento a los fenicios gaditanos.

Por otra parte, esta ausencia de planteamiento científico en la explicación del fenómeno se inscribe perfectamente en la tradición posidoniana, en la medida en que ha llegado hasta nosotros. Posidonio, en efecto, es un sabio muy pragmático. Su estudio de los fenómenos anormales no sólo se caracteriza por un retorno a la antigua

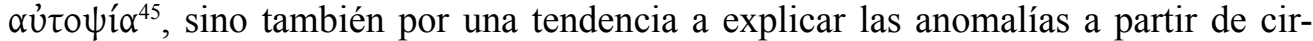
cunstancias particulares. Para Posidonio, los fenómenos anormales ni son tales, ni requieren una integración en el sistema cosmológico. Son el resultado de circuns-

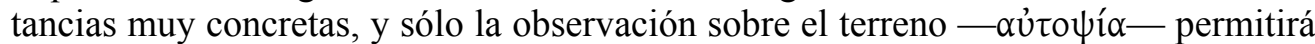
al científico avisado descubrir el «truco». Esta forma de actuar aparece reflejada en otros dos textos relacionados con Gadira, lugar que visitó en persona: su refutación de las anomalías de las puestas de sol en Occidente ${ }^{46}$, y la localización de las Columnas de Heracles ${ }^{47}$. En el primer caso, la creencia de que el sol se ponía repentinamente en Occidente, corriente en el mundo grecorromano, se explica de esta forma: örou

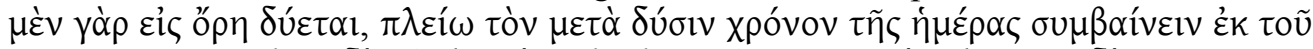

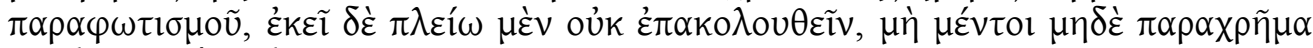

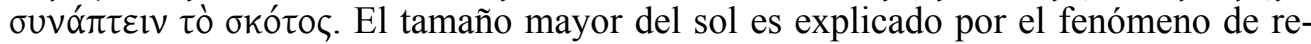

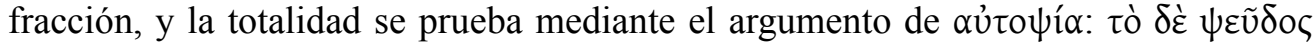

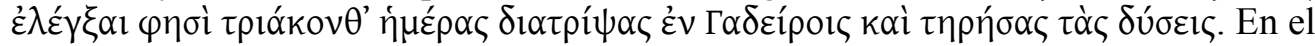
caso de las Columnas de Heracles, frente a los argumentos geográficos y mitológicos, Posidonio postula que las Columnas son... columnas, las columnas del templo, y que los que han llegado hasta allí y visitado el recinto sagrado han extendido, en beneficio propio, la falsa idea de que allí se encuentra el fin del mundo. Si, como piensa Kidd ${ }^{48}$, la historia de la fundación de Cádiz según los gaditanos que forma parte del mismo pasaje ha sido tomada por Estrabón de la obra de Posidonio, es posible que la descripción las columnas del templo y la teoría que las identifica con las Columnas sea parte de una crónica de la visita al templo del filósofo de Apamea, donde se haría hincapié en su comprobación in situ.

De este modo, podemos observar una estructura recurrente, referente a debates científicos cuyo objeto Posidonio estudió en persona, y sobre los que elaboró teorías destinadas a proporcionar una explicación. En los tres casos, en lugar de aceptar sin

\footnotetext{
${ }^{44}$ Str. 3.5.7.

${ }^{45}$ PAJón LeYra (2009: 496).

${ }^{46}$ Str. 3.1.5.

${ }^{47}$ Str. 3.5.5-6.

${ }^{48}$ KIDD (1988: 846-851).
} 
más una tradición existente, Posidonio acude al lugar, se informa, y llega a la conclusión de que la tradición ha surgido de una confusión generalizada, con o sin intención, por gente sin conocimientos científicos. Detrás de esta confusión no hay una teoría complicada que requiera grandes explicaciones, sino un fenómeno de lo más habitual, o un objeto de lo más ordinario.

Esto, sin embargo, no quiere decir que estos fenómenos ordinarios de Posidonio no se encuentren regulados a su vez por verdaderas teorías científicas, cuya recurrencia en el cosmos no las hace menos señaladas. En este caso, el error de los gaditanos se encuentra relacionado con las mareas, cuyo comportamiento estudió, con gran éxito, el filósofo durante su visita a la ciudad atlántica. De hecho, la teoría de las mareas de Posidonio, expuesta por Estrabón en un excurso al $\pi \alpha \rho \alpha ́ \delta \circ \xi o v$, aparece expuesta no sólo aquí, sino en diversos otros textos de la Antigüedad ${ }^{49}$, y gozó de un gran predicamento. Tal teoría consiste, a grandes rasgos, en el establecimiento de tres ciclos: el diurno; el mensual y el anual, relacionados con la posición de la luna en el cielo. Los dos precursores más señalados de Posidonio en este campo son Píteas, a quien se atribuye el descubrimiento de la relación de la luna con las mareas ${ }^{50}$, y Seleuco, también citado por Estrabón en este pasaje como un estudioso de los ciclos anuales en el Mar Rojo ${ }^{51}$. La idea de que la luna, desde el cielo, era capaz de afectar el comportamiento de las aguas constituyó un fuerte apoyo a la idea de simpatía universal defendida por el estoicismo.

Por último, Estrabón nos hace partícipes de dos teorías, una de ellas de su propia cosecha, y la segunda basada en conceptos establecidos por Atenodoro, también experto en las mareas. La primera introduce el concepto del agua, normalmente considerada pasiva, como agente debilitador, que hace que las paredes del conducto de la fuente se vuelvan mullidas y porosas y que el agua de la fuente escape a través de ellas en lugar de seguir su camino hacia la superficie. Esta idea aparece en otros lugares de la literatura científica, como Str. 1.3.5, donde sirve para explicar la causa de los terremotos. Al afectar de esta manera a la tierra, el agua la vuelve vulnerable

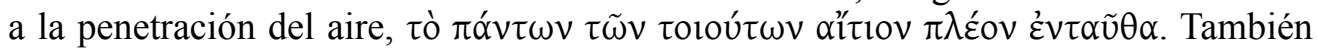
Sen.nat. 6.15.1, al explicar las causas de los terremotos, señala al agua como agente responsable ${ }^{52}$. Según Séneca, el aire que penetra en la tierra a través de los intersticios formados por la acción del agua también es encerrado por las olas, pero su reacción es más violenta que en el caso de la fuente, y provoca la ruptura de la tierra. En nat. 6.22.4 se atribuye esta teoría a Calístenes, filósofo y sobrino de Aristóteles.

En cuanto a la teoría de Atenodoro, su premisa yace en la hipótesis de que las ma-

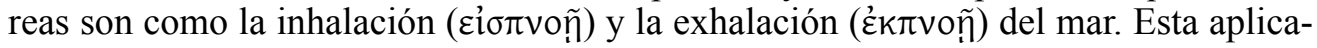
ción de conceptos biológicos al mundo físico es una característica de la ciencia griega

${ }^{49}$ Sen. nat. 3.28.6, Plin.nat. 2.215, y Prisc.Lyd. Solut.6, p. 71 Bywater.

${ }^{50}$ Diels (1879: 383). Cf. Roseman (1994: 102-103.)

${ }^{51}$ Str. 3.5.9.

${ }^{52}$ Terra multis locis perforata est nec tantum primos illos aditus habet quos velut spiramenta ab initio sui recepit, sed multos illi casus imposuit. Aliubi deduxit quicquid superne terreni erat aqua, alia torrentes cecidere, alia aestibus magnis disrupta patuerunt. Per haec intervalla intrat spiritus. Quem si inclusit mare et altius adegit nec fluctus retro abire permisit, tunc ille, exitu simul redituque praecluso, volutatur et, quia in rectum non potest tendere, quod illi naturale est, in sublime se intendit et terram prementem diverberat. 
desde sus orígenes. Aristóteles nos cuenta que ya Empédocles había comparado la tierra a un ser vivo, y el mar a su sudor ${ }^{53}$. Ovidio atribuye la misma doctrina a Pitágoras ${ }^{54}$. Aristóteles compara los terremotos con los escalofríos que sacuden el cuerpo después de que la orina lo abandone ${ }^{55}$, y según Diodoro, Éforo habría declarado que la crecida del Nilo es el sudor de la tierra de Egipto en verano ${ }^{56}$.

Por su parte, Séneca se hace eco de estas ideas relacionándolas con el caso concreto de los conductos subterráneos de agua, es decir, de los manantiales, explicando así el

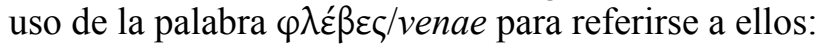

Placet natura regi terram, et quidem ad nostrorum corporum exemplar, in quibus et venae sunt et arteriae, illae sanguinis, hae spiritus receptacula. In terra quoque sunt alis itinera per quae aqua, alia per quae spiritus currit; adeoque ad similitudinem illa humanorum corporum natura formavit ut maiores quoque nostri aquarum apellaverint venas. (SEN.nat.3.15.1)

Este uso lo encontramos en nuestro pasaje:

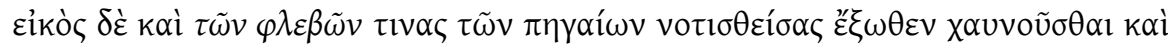

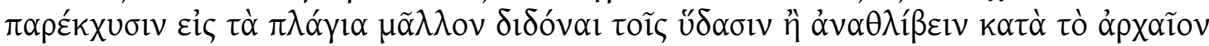

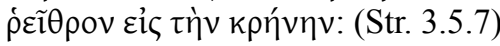

La idea de inhalación y exhalación de Atenodoro, sin embargo, comparando al mar en concreto con un organismo vivo, y las mareas con su respiración, nos remite principalmente a Str. 1.3.8:

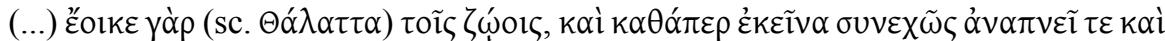

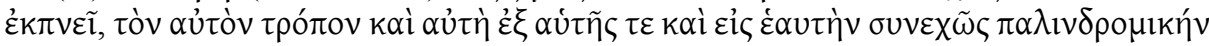

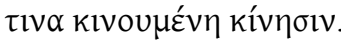

También otros autores, como Solino y Mela, se hacen eco de las teorías de inhalación y exhalación:

Neque adhuc satis cognitum est, anhelitune id suo mundus efficiat, retractamque cum spiritu regerat undam undique, si, ut doctioribus placet, unum animal est, an sint depressi aliqui specus, quo reciprocata maria residant, atque unde se rursus exuberantia adtollant, an luna causas tantis meatibus praebeat. (MelA.3.2)

\footnotetext{
${ }^{53}$ Arist. Mete 2. 353b.

${ }^{54}$ Ov. met. 15.342 y ss. Cf. Oltramare (1961: 129).

${ }^{55}$ Arist. Mete. 2.366b.

${ }^{56}$ D.S. 1.39 .7$.
} 
Sicut ergo in corporibus nostris commercia sunt spiritalia, ita in profundis oceani nares quasdam mundi constitutas, per quas emissi anhelitus vel reducti modo efflent maria, modo revocent (...) (SoL. 23.21.57)

La idea de la inhalación y la exhalación del mar, a su vez, se relaciona con otros dos conceptos científicos. Uno de ellos aparece formulado tanto en el texto de Mela como en el nuestro: la idea del agua como una masa oscilante que se introduce a través de una serie de conductos conectados entre sí, dando lugar a las mareas, los ríos o las fuentes según la dirección que haya tomado. La idea se encuentra presente ya en el Fedón, donde el movimiento de unos conductos a otros es comparado con los movimientos de la respiración animal:

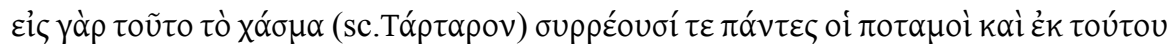

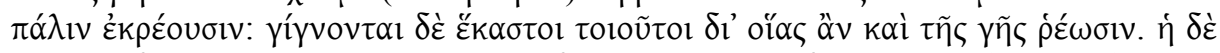

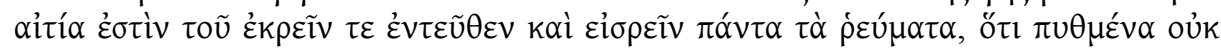

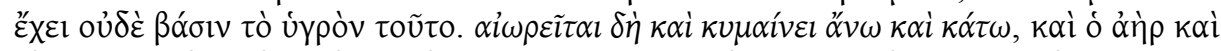

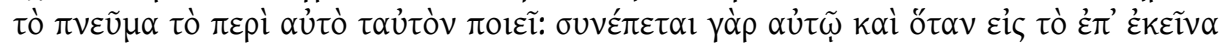

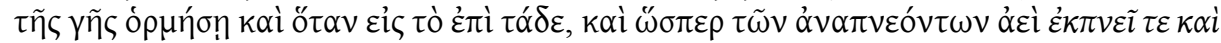

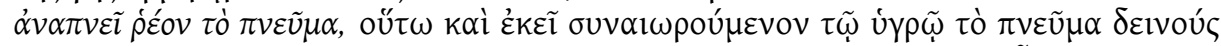

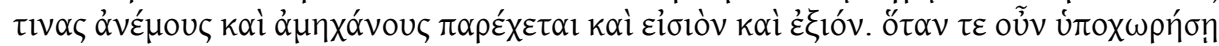

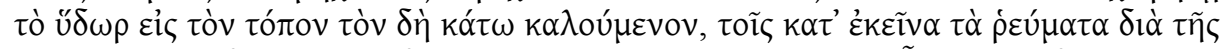

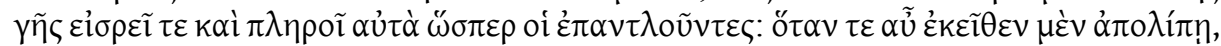

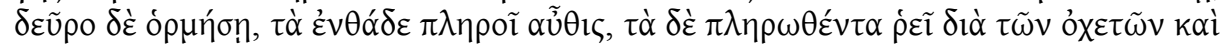

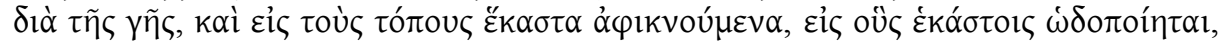

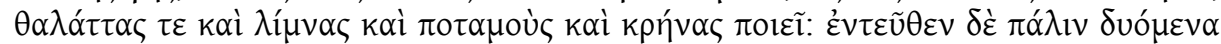

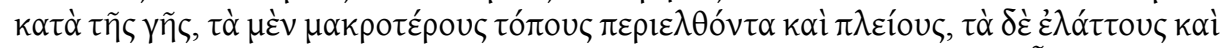

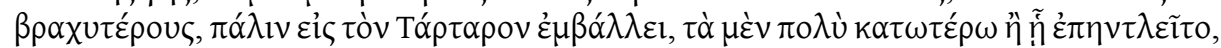

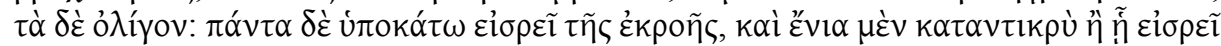

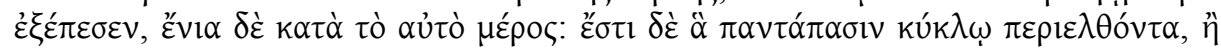

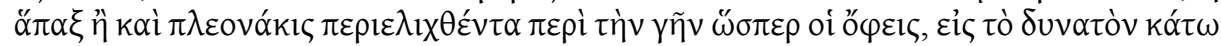

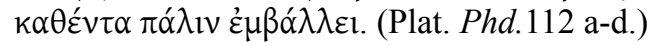

Atenodoro/ Estrabón postulan un fenómeno similar en el ámbito restringido de la fuente y la marea. El agua del mar y la de la fuente se encuentran interconectadas por conductos subterráneos, y el agua oscila de unos a otros siguiendo el ritmo de la inhalación y exhalación ${ }^{58}$.

${ }^{57}$ Existe incluso la posibilidad de que Píteas de Marsella, el científico y navegante que «recorrió la costa de Europa desde Gadira hasta el Tanais» (Str. 2.4.1), se hubiera referido a esta teoría. En T30 Roseman (=Schol. in A.R., $4.761-5$ a) podemos leer que Píteas afirmó que el mar hierve. Según el co-

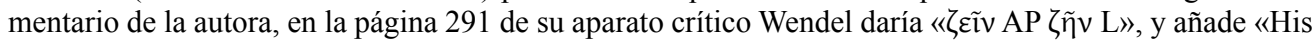
(Wendel's) own discussions of the MSS in Scolia Apollonium Rhodium vetera (Berlin 1958) and in RE make it clear that $\mathrm{L}$ is the best manuscript both for the text of Apollonios and the accompanyng scholia. There is some possibility, then, that $\zeta \tilde{\eta} v$ stood in the original source used by early commentators. One would then translate "saying also that the sea is alive..."». Roseman (1994: 114-115).

${ }^{58}$ Str. 3.5.7. 
Por su parte Filóstrato, en una época más tardía, presenta la versión de Apolonio de Tiana — que también, imitando a sus ilustres antecesores, acudió a Cádiz a observar el fenómeno- sobre esta teoría:

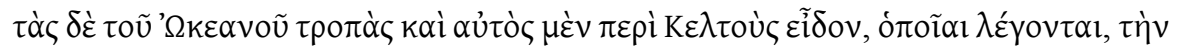

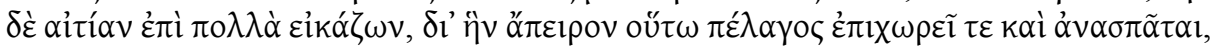

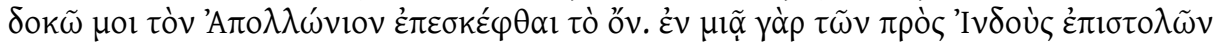

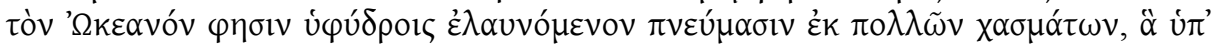

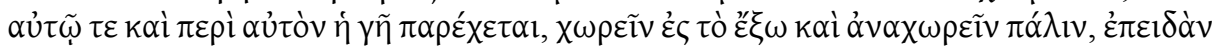

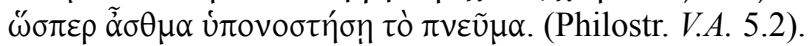

El texto, sin embargo, continúa, pasando de ésta al segundo concepto científico relacionado con la respiración del océano:

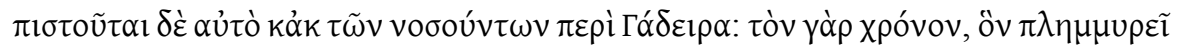

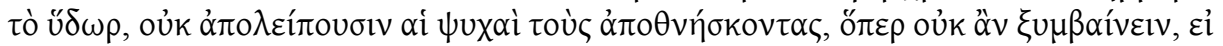

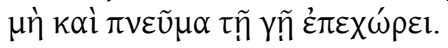

Tal idea es mucho más antigua que Filóstrato, y se retrotraería hasta el propio fundador del Liceo. Según nos dice Plinio:

his addit - ut nihil, quod equidem noverim, praeteream - Aristoteles nullum animal nisi aestu recedente expirare. observatum id multis in Gallico oceano et dumtaxat in homine compertum. (Plin. nat. 2.220)

Se trata, pues, de una tradición cuyo origen hunde sus raíces en el concepto de

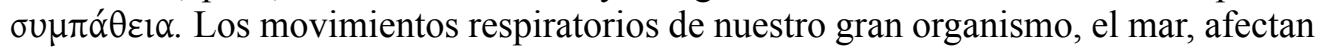
a organismos menores que se encuentran en una situación de proximidad con respecto a él: los humanos y los animales. Esta influencia adquiere la forma de una atracción, y un paralelo exacto se establece entre, por una parte, la atracción de las aguas hacia sus hendiduras a causa del empuje de la inhalación, y por otra la atracción de las almas (entendidas como una substancia corpórea compuesta generalmente de agua y fuego), que abandonan los cuerpos. De este modo, la respiración del mar no sólo afectaría a todo aquello que está físicamente conectado a éste, como la fuente a través de los conductos subterráneos, sino que, al igual que la influencia de la luna sobre las mareas, también se dejaría sentir en forma de influjo sobre aquello que se encuentra unido por

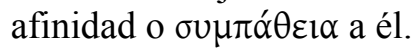

\section{CONCLUSIONES}

A pesar de encontrarse inserto en un catálogo de curiosidades relacionadas con la descripción geográfica de una ciudad, el texto estraboniano sobre la fuente del Heracleo refleja la existencia de un verdadero debate científico sobre el fenómeno. Estrabón menciona los nombres de Artemidoro, Sileno, Polibio, Posidonio y Atenodoro como 
parte de este debate, y procede a exponer y criticar sus respectivas opiniones, proponiendo otras de su propia cosecha. Todos, en general, pretenden demostrar que existe una explicación racional que ancla el supuesto $\pi \alpha \rho \alpha ́ \delta \circ \xi o v$ a las leyes generales de la ciencia tal y como se las entendía en aquella época, procedimiento opuesto al habitualmente seguido por los paradoxógrafos, que aíslan el fenómeno de su contexto científico para aumentar la sensación de maravilla de su audiencia. Para la ciencia estoica, en efecto, los sucesos paradoxográficos como tales no tienen cabida en el mundo, y la maravilla no es sino una manifestación de ignorancia de las verdaderas causas. El

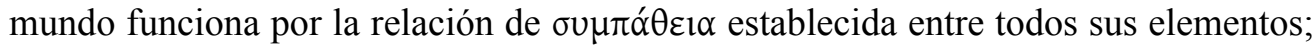
y cualquier rotura aparente de este orden universal debe ser investigada y sus causas reales expuestas.

Pero no todas las teorías son del mismo tipo. Posidonio, por ejemplo, se desmarca un tanto del resto al intentar demostrar que la pretendida relación de ảv debe a ninguna causa científica, sino a un error de percepción de los locales. Polibio, sin embargo, proporciona una explicación donde entran en juego diversos conceptos de la ciencia helenística en general y estoica en particular, como el aire como motor principal de los procesos naturales (como es el caso de los terremotos), y el encadenamiento de las causas provocado por la ausencia de vacío (concepto ya presente en la filosofía de Platón.) La explicación del propio Estrabón, a su vez, se basa en un concepto generalmente aceptado, el del agua como agente debilitador. Finalmente, se invoca la teoría de Atenodoro, según la cual la marea es como la «respiración» del mar, una «biologización» de los fenómenos del mundo físico corriente en la ciencia griega desde sus orígenes. Esto, a su vez, nos conduce a una noción presente ya en el Fedón, que presenta al agua como una masa oscilante que corre a través de una serie de conductos subterráneos conectados entre sí al ritmo de la respiración animal, dando lugar a las distintas manifestaciones acuáticas observables. La idea del Océano como un «gran

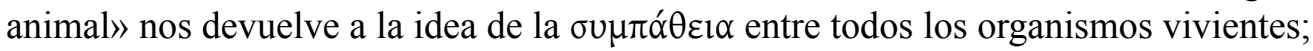
en este caso entre un gran organismo (el Océano) y los organismos menores que se encuentran próximos a él, como los animales y las personas - de donde proceden, a su vez, las diversas noticias sobre el comportamiento simpático con las mareas de las almas de los seres vivos en zonas próximas al Océano como Cádiz.

La presencia de todos estos nombres ilustres y teorías especializadas demuestran la importancia de la fuente gaditana como objeto de discusión de la ciencia, no ya sólo como una anécdota curiosa a incluir en las listas de los paradoxógrafos, sino como un verdadero problema que ponía a prueba el ingenio individual del científico, y lo obligaba a ejercer una labor crítica con respecto a los esfuerzos de sus predecesores.

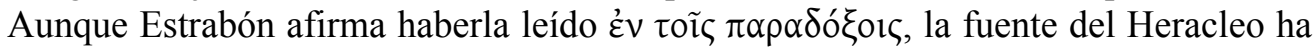
trascendido la naturaleza de mera curiosidad para convertirse, al igual que la fuente de Amón, en un lugar común establecido de la ciencia helenístico-imperial. 


\section{BiBLIOGRAFÍA}

Bianchetti, Serena (2008): «Geografia e cartografia dell'estremo occidente. Da Eratostene a Tolomeo», Mainake 30: 17-58.

Bianchetti, Serena (1997): «Conoscenze geografiche e rappresentazioni dell'ecumene nell'antichità greco-romana», en C. Tugnoli (ed.), I contorni della terra e del mare. La geografia tra rappresentazione e invenzione della realtà. Bologna: 51-92.

Clarke, Katherine (1999), Between geography and history: Hellenistic constructions of the Roman world. Oxford.

Diels, Hermann A. (1879, reimp. 1958), Doxographi Graeci. Berlin.

Giannini, Alessandro (1965), Paradoxographorum Graecorum Reliquiae. Milano.

Irby-Massie, Georgia L., Keyser, Paul T. (2002), Greek Science of the Hellenistic Era. London-New York.

KIDD, Ian G. (1988), The Fragments of Posidonius part II: The Commentary. Cambridge University Press.

Lloyd, Geoffrey E.R. (1973), Greek Science After Aristotle. New York.

Oltramare, Paul (1961), Sénèque. Questions Naturelles, Tome 1. Paris.

Pajón Leyra, Irene. (2009), Paradoxografía griega: estudio de un género literario. Madrid.

PÉDECH, Paul (1956), «La géographie de Polybe: structure et contenu du livre XXXIV des Histoires», LEC 24: 3-24.

PÉdech, Paul (1964), La méthode historique de Polybe. Paris.

Peretti, Aurelio (1979), Il Periplo di Scilace: Studio sul primo portolano del Mediterraneo. Pisa.

Romm, James S. (1992), The Edges of Earth in Ancient Thought. Princeton.

Roseman, Christina H. (1994), Piteas of Massalia. On the Ocean: Text, translation and commentary. Michigan.

Sambursky, Samuel (1959), Physics of the Stoics. New York.

Schepens, Guido, Delcroix, Kris (1996), «Ancient Paradoxography: Origin, Evolution, Production and Reception», en Pecere \&Stramaglia (eds.), La letteratura di consumo nel mondo greco-latino. Casini.

Vattuone, Roberto (2002), «Timeo di Tauromenio», en R.Vattuone (ed.), Storici greci di Occidente. Bologna.

Walbank, Frank W. (1972), Polybius. Berkeley. 